\title{
Rayonnement UVX créé par génération d'harmoniques d'un champ laser intense
}

\author{
A. L'Huillier, P. Salières et Ph. Balcou(1) \\ Service des Photons, Atomes et Molécules, Centre d'Etudes de Saclay, 91191 Gif-sur-Yvette, \\ France
}

Résumé : Nous decrivons les processus de génération d'harmoniques d'ordre élevé dans les gaz rares: la compréhension du phénomène physique, les caractéristiques du rayonnement, et les premières applications en spectroscopie atomique.

\begin{abstract}
We describe high order harmonic generation processes in rare gases: the understanding of the physical phenomena, the characteristics of the radiation and the first applications in atomic spectroscopy.
\end{abstract}

\section{INTRODUCTION}

Lorsqu'un laser intense est focalisé dans un jet de gaz rare, des harmoniques d'ordre très élevé sont générées. L'harmonique 109 d'un laser de type Titane-Saphir de longueur d'onde $806 \mathrm{~nm}$, ou bien l'harmonique 135 d'un laser à verre dopé au Néodyme de longueur d'onde $1053 \mathrm{~nm}$ ont été observées. Ce rayonnement se présente sous forme de raies aux fréquences harmoniques impaires du laser incident, avec des intensités presque constantes.

Le plan de ce manuscrit est le suivant. Après une brève description de la méthode expérimentale, nous discutons les principaux résultats obtenus et la compréhension du phénomène physique. Puis nous donnons les caractéristiques du rayonnement, pour les applications potentielles.

\section{METHODE EXPERIMENTALE}

Un schéma expérimental typique $[2,3]$ pour ces expériences est indiqué sur la figure 1 . La pression dans la région d'interaction à la sortie du jet de gaz est de quelques dizaines de Torr. Le rayonnement produit dans l'axe de propagation du laser est analysé à l'aide d'un couple miroir torique/réseau plan qui permet à la fois de refocaliser le rayonnement spatialement et de le disperser spectralement pour séparer les différentes harmoniques. Les harmoniques sont ensuite détectées par un multiplicateur d'électrons secondaires, alors que le rayonnement incident refléchi par le réseau est piégé.

(1) Adresse actuelle : Laboratoire d'Electronique Quantique, UA 1202 du CNRS, Université de Rennes 1, 35042 Rennes, France 


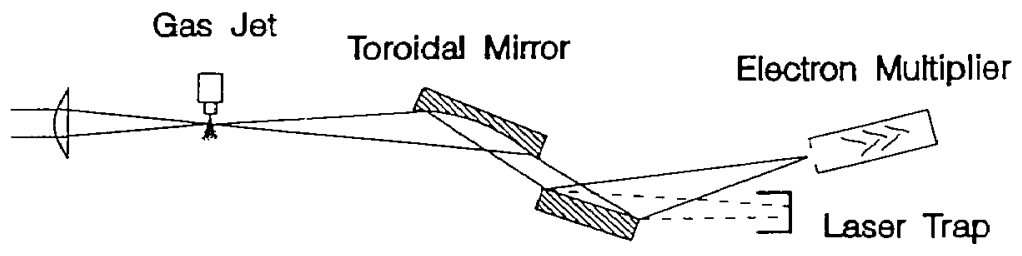

Grating

Figure 1 : Schéma expérimental typique pour la détection du rayonnement harmonique.

On peut aussi utiliser d'autres détecteurs : caméra à balayage de fente qui permet d'avoir des informations concernant la durée des différentes harmoniques, ou galettes de microcanaux (couplées à un écran phosphore et une caméra $\mathrm{CCD}$ ) pour imager la distribution spatiale des harmoniques. Toutes ces techniques apportent des connaissances complémentaires sur les caractéristiques du rayonnement.

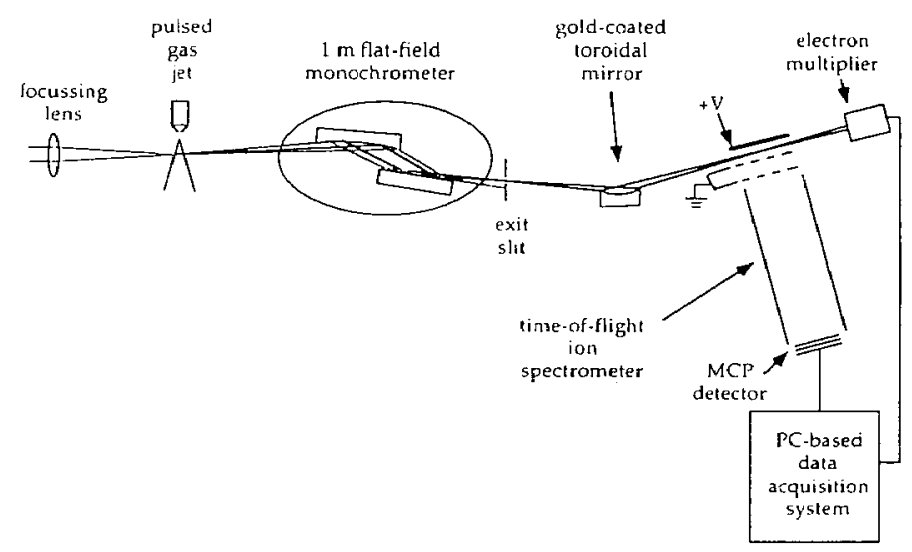

Figure 2: Dispositif expérimental pour une application du rayonnement en spectroscopie atomique.

Finalement, une première expérience d'application du rayonnement a été réalisée [4]. Le dispositif expérimental est présenté sur la figure 2 . Un deuxième miroir torique refocalise le rayonnement dans un enceinte d'application. Dans cette expérience, les ions produits à la suite de l'interaction avec le rayonnement harmonique sont détectés par un spectromètre à temps de vol.

\section{PRINCIPAUX RESULTATS}

La figure 3 montre un résultat typique obtenu sur du xénon avec un laser de type verre dopé au Néodyme [2] de durée $1 \mathrm{ps}$, de longueur d'onde $1053 \mathrm{~nm}$ et d'intensité $10^{14}$ $\mathrm{W} / \mathrm{cm}^{2}$. Après la décroissance attendue pour les ordres 3 et 5 , l'intensité des harmoniques reste pratiquement constante jusqu'à la 23 ième harmonique environ, avant de décroître à nouveau brusquement. L'éclairement laser est suffisamment intense pour induire une réponse non-perturbative de l'atome au champ électromagnétique, avec pour conséquence cette distribution caractéristique. 


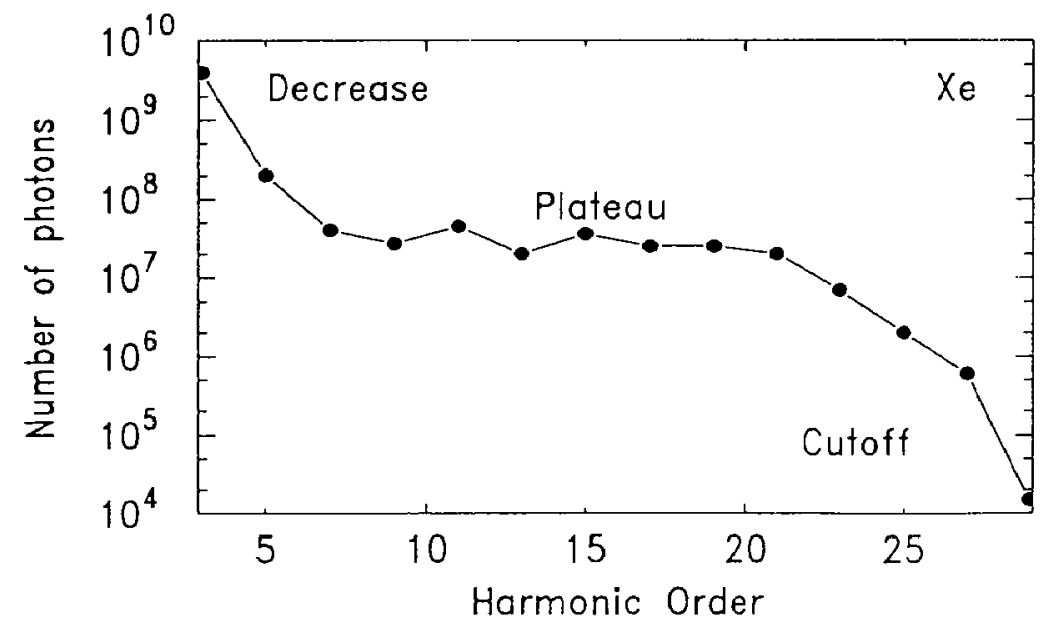

Figure 3 : Spectre d'harmoniques obtenu sur du xénon. Les caractéristiques du laser incident sont : $1 \mathrm{ps}, 1053 \mathrm{~nm}$ et $10^{14} \mathrm{~W} / \mathrm{cm}^{2}$.

Si l'on utilise des gaz rares plus légers, comme le néon ou l'hélium, on peut aller beaucoup plus loin vers les courtes longueurs d'onde. La figure 4 résume les résultats obtenus sur les quatre gaz rares $\mathrm{Xe}, \mathrm{Ar}, \mathrm{Ne}$ et He [3]. Les harmoniques les plus élevées

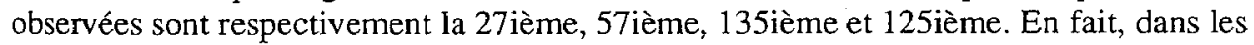
2 derniers cas, on atteint la limite du spectromètre. L'échelle verticale est une estimation du nombre de photons obtenu à chaque tir laser. Si l'on va beaucoup plus loin en énergie dans le cas des gaz rares légers, l'efficacité de conversion dans la région du plateau est cependant plus faible.

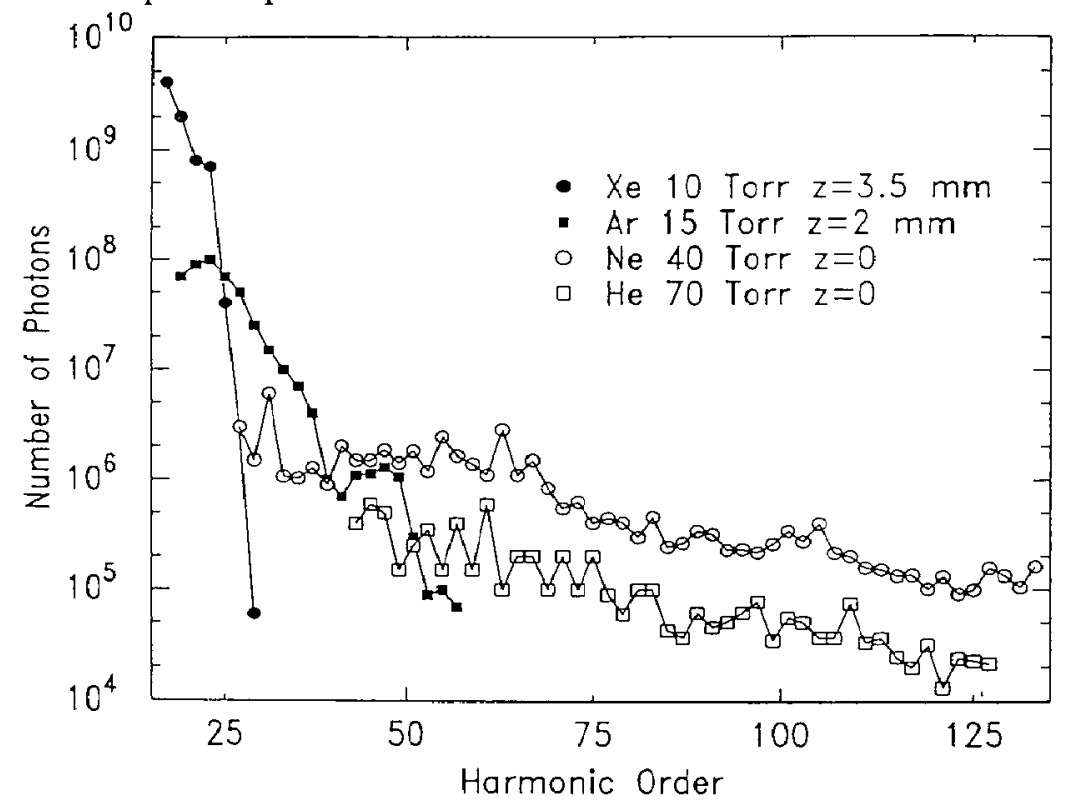

Figure 4. Spectre d'harmoniques obtenu dans le xénon, argon, néon et hélium avec un laser à verre dopé au Néodyme. L'intensité utilisée est de l'ordre de $10^{15} \mathrm{~W} / \mathrm{cm}^{2}$ 
Finalement, la figure 5 présente un résultat obtenu avec un laser de type Titane-Saphire $(800 \mathrm{~nm}, 150 \mathrm{fs})$, qui possède une cadence de répétition élevée $(10 \mathrm{~Hz})$ et qui est probablement le laser le plus adapté pour une géneration d'harmoniques efficace [5]. Ici, nous montrons comment les harmoniques successives varient en fonction de l'intensité laser. Après une dépendance rapide qui correspond à la région de la coupure, les harmoniques joignent le plateau. La dépendance est alors assez lente et similaire pour toutes les harmoniques.

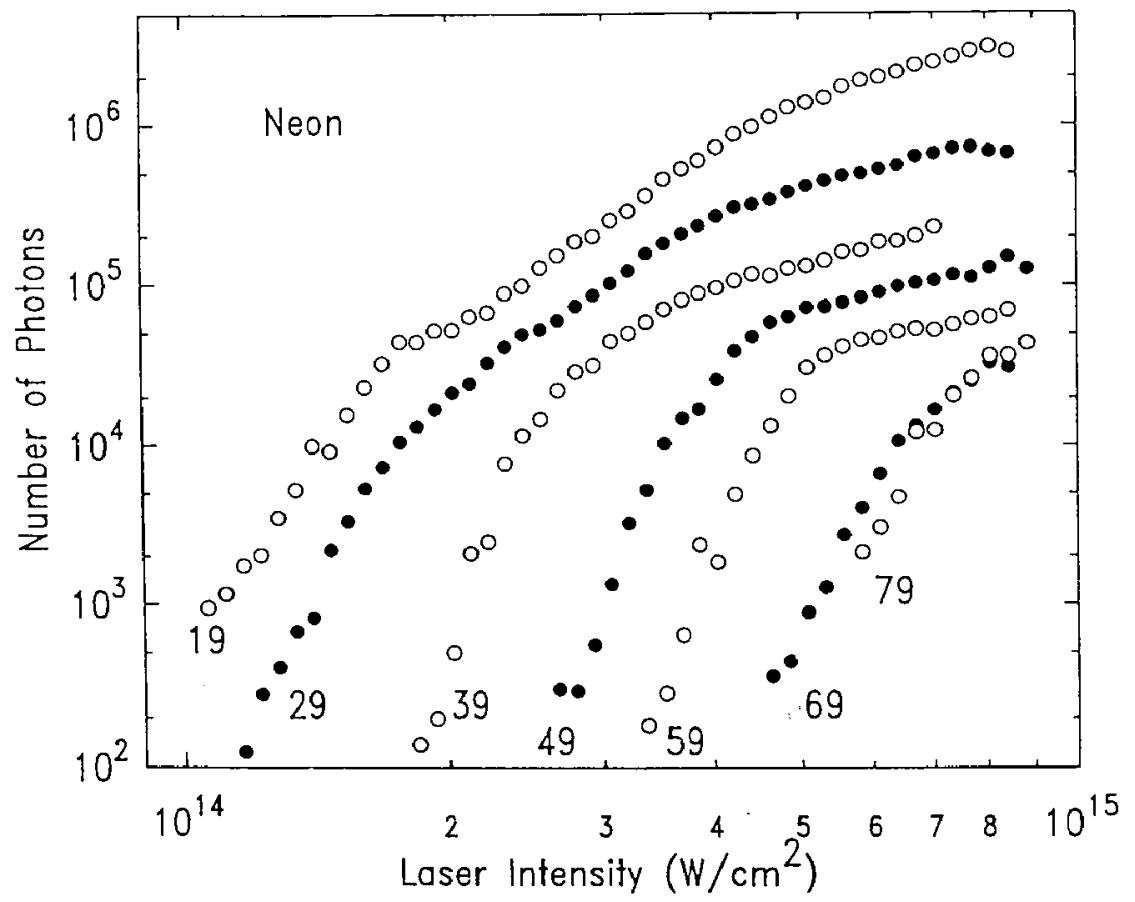

Figure 5: Dépendance des harmoniques 19 à 79 générées dans du néon en fonction de l'intensité du rayonnement incident (250 fs, $794 \mathrm{~nm}, 10 \mathrm{~Hz}$ ).

L'interprétation théorique de ces expériences comporte deux étapes :

1) le calcul de la réponse d'un atome à un champ laser intense (domaine de la physique atomique).

2) la résolution des équations de propagation pour les champs harmoniques créés dans le milieu (domaine de l'optique nonlinéaire).

Ces deux étapes nécessitent des calculs numériques assez poussés qu'il serait trop long de détailler dans ce manuscrit, et qui donnent, en général un accord théorie-expérience satisfaisant. Les simulations numériques ont en particulier permis de dégager une loi d'échelle importante : celle de la position en énergie de la coupure dans le spectre d'harmoniques [6]. L'énergie maximum atteinte à la fin du plateau est $W_{p}=I_{p}+3 U_{p}$, où $I_{p}$ est l'énergie d'ionisation de l'atome et $U_{p}$, l'énergie d'oscillation d'un électron dans le champ laser, proportionnclle à l'intensité du champ laser et à l'inverse du cuaré de la fréquence laser. Cette loi n'est valable que jusqu'à l'intensité à laquelle l'atome s'ionise. Elle montre qu'il faut utiliser de préférence des gaz rares légers qui possèdent une énergie d'ionisation élevée et ne s'ionisent pas facilement, ainsi qu'un laser de basse fréquence pour produire des photons d'énergie importante. Les résultats 
expérimentaux confirment qualitativement cette loi de coupure, et en particulier les dépendances en fonction de l'intensité ou de la fréquence. L'énergie maximum observée expérimentalement pour un atome et une intensité laser donnés se trouve être légèrement plus faible que celle prédite par la loi de coupure, ce qui peut s'expliquer par des effets dus à la propagation dans le milieu gazeux.

Une interprétation physique simple du phénomène de génération d'harmoniques d'ordre élévé a pu également être dégagée [7]. Dans un régime où la fréquence du laser excitateur est faible devant l'énergie d'ionisation, la génération d'harmoniques a lieu en trois étapes : ionisation de l'électron par effet tunnel, accéleration dans le champ laser, puis recombinaison vers l'état fondamental si la trajectoire classique de l'électron passe dans le voisinage du potentiel atomique.

\section{CARACTERISTIQUES DU RAYONNEMENT HARMONIQUE ET APPLICA- TIONS.}

Abordons un aspect plus technique : quelles sont les caractéristiques du rayonnement XUV produit dans la génération d'harmoniques ? en vue de quelles applications ? Ces caractéristiques dépendent évidemment de celles du laser de pompe. Nous discutons essentiellement celles obtenues avec un laser Titane-Saphire, le plus adapté à l'heure actuelle pour la génération d'harmoniques et leur utilisation. Le rayonnement harmonique est comparable à un rayonnement, avec, dans certaines conditions, des propriétés semblables de cohérence spatiale ou temporelle.

\subsection{Durée d'impulsion.}

La durée des impulsions harmoniques générées est un peu inférieure à celle du laser de pompe, typiquement entre 50 et 100 femtosecondes, si l'on part d'un laser de durée 150 fs. C'est donc un rayonnement de durée très courte (et donc de grande puissance instantannée). Notons toutefois un petit problème technique inhérent aux techniques expérimentales utilisées jusqu'à présent. Le réseau d'analyse introduit un élargissment temporel de l'ordre de plusieurs picosecondes. Il devrait être possible d'utiliser un deuxième réseau compensant cet effet.

Cet aspect du rayonnement harmonique "ultracourt" permet d'envisager des expériences de type pompe-sonde dont le but est d'étudier une dynamique temporelle sur une échelle de temps de l'ordre de $100 \mathrm{fs}$ (ou plus). L'idée est d'exciter par exemple une surface ou une molécule, et de sonder l'effet de cette excitation à l'aide d'un deuxième pulse dont le retard par rapport au premier peut être ajusté. Les deux pulses peuvent être de "couleur" différente (laser + harmonique, ou harmonique + laser, ou 2 pulses harmoniques). Si ces techniques sont abondamment utilisées dans le domaine visible, elles n'ont jamais été envisagées dans le domaine XUV, faute de source de durée suffisamment courte.

\subsection{Largeur spectrale}

Contrairement aux idées habituelles sur les lasers, la résolution spectrale n'est pas extraordinaire, parce que l'on utilise des lasers de courte durée d'impulsion. Le principe d'Heisenberg : $\Delta \mathrm{t} \Delta v=\mathrm{Cte}$, implique que plus la durée du rayonnement est courte, moins il sera monochromatique. Ainsi, la largeur spectrale d'une harmonique d'un laser de durée $150 \mathrm{fs}$ est de l'ordre de l'Angström. Typiquement, nous avons mesuré une résolution $\Delta v / v$ de l'ordre de $3 \times 10^{-3}$ vers $40 \mathrm{eV}$.

Pour les applications demandant une meilleure résolution spectrale, il est possible d'utiliser des lasers de durée plus longue, quelques dizaines de picosecondes. On 
dispose alors d'une résolution spectrale de l'ordre de $10^{-5}$ vers $20 \mathrm{eV}$, bien meilleure que ce qui se fait actuellement avec les meilleurs monochromateurs. Bien sûr, il ne sera pas possible d'aller aussi loin en énergie avec ces lasers, mais il semble que cette caractéristique du rayonement harmonique (résolution spectrale) intéresse une grande communauté scientifique en physique atomique et moléculaire.

\subsection{Accordabilité}

Pour une fréquence fixe du laser incident, le rayonnement harmonique se présente sous forme de raies séparées de deux fois l'énergie d'un photon laser (donc de $3 \mathrm{eV}$ dans le cas d'un laser Ti:S) qui peuvent être sélectionnées tour à tour. Certaines applications peuvent demander une accordabilité plus fine. Les lasers de type Titane Saphire peuvent en principe être accordés entre 720 et $900 \mathrm{~nm}$ mais pas facilement, le changement de longueur d'onde nécessitant un réalignement complet. Les lasers à colorants sont plus faciles à accorder. On peut aussi envisager le mélange de plusieurs fréquences permettant d'atteindre un domaine spectral plus étendu. En résumé, si le rayonnement harmonique n'est pas continûment accordable pour l'instant sur toute l'étendue spectrale accessible (10-150 eV), il est néanmoins possible d'atteindre n'importe quelle énergie, en jouant sur la longueur d'onde du laser de pompe.

\subsection{Distribution angulaire et cohérence spatiale}

Le rayonnement harmonique est émis dans l'axe du laser sur un cône de divergence très faible. Nous présentons sur la figure 6 les profils d'émission de quelques harmoniques $(23,25,27,29)$ générées dans l'argon à l'aide d'un laser de type LiSAF dont les caractéristiques sont proches de celles d'un Titane-Saphire [8]. La divergence du laser incident est égale à $55 \mathrm{mrad}$ à mi hauteur. La divergence des harmoniques varie de 5 mrad pour l'harmonique 29 dans la région de la coupure à environ $13 \mathrm{mrad}$ pour l'harmonique 23 dans la région du plateau. Les profils sont symétriques et sans structure. Ces résultats sont très encourageants pour certaines applications du rayonnement harmonique. Avec des optiques convenables, par exemple, des zones de Bragg-Fresnel, il devrait être possible de focaliser ce rayonnement sur des taches très petites.

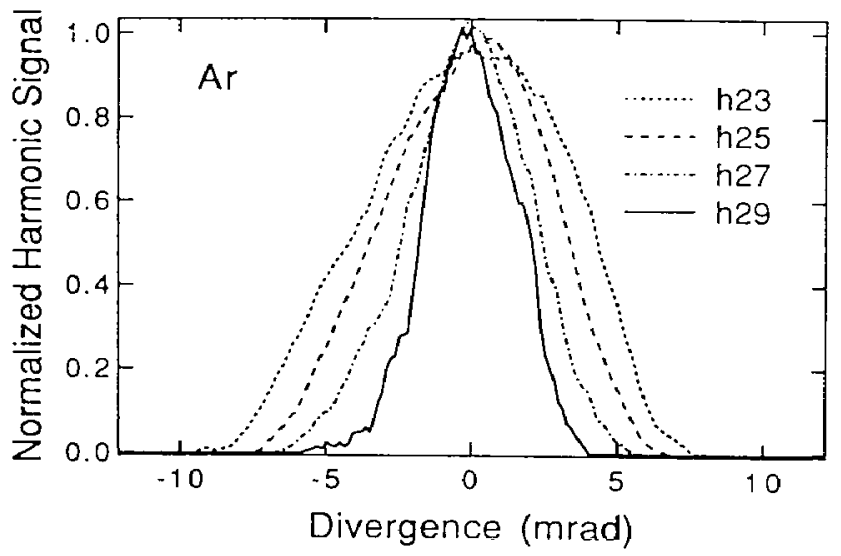

Figure 6 : Distributions angulaires pour quelques harmoniques générées dans de l'argon avec un laser LiSAF. 


\subsection{Nombre de photons}

Une caractéristique importante du rayonnement harmonique est le nombre de photons émis à chaque tir laser (à partir duquel on peut calculer la puissance moyenne ou instantannée, l'énergie, etc..). Il dépend de façon importante du gaz utilisé pour la génération d'harmoniques ainsi que des conditions géométriques de l'interaction, en particulier, le degré de focalisation du laser incident (moins celui-ci est focalisé, plus le rendement de conversion est bon, à condition bien sûr que l'intensité dans la région d'interaction reste la même). Des conditions typiques d'expérience sont environ $10^{9}$ photons dans une gamme spectrale de 10 à $40 \mathrm{eV}$, utilisant des gaz lourds (xénon, argon) et $10^{6}-10^{7}$ photons de 40 à $150 \mathrm{eV}$, où il faut utiliser des gaz légers (néon, hélium).

Pour terminer cette communication, nous décrivons une première expérience de spectroscopie réalisée avec le rayonnement harmonique.

\subsection{Expérience de spectroscopie atomique}

Le dispositif expérimental [4] a été décrit ci-dessus (voir la figure 2). Un miroir torique refocalise le rayonnement harmonique dans une enceinte d'application. La cible gazeuse est fournie par un jet pulsé permettant d'atteindre une pression de $10^{-3} \mathrm{Torr}$ dans la région d'interaction. Les ions produits sont analysés à l'aide d'un spectromètre à temps de vol et détectés par des galettes de microcanaux. Un multiplicateur d'électrons secondaires, placé dans l'axe de la propagation des harmoniques, permet de mesurer un signal proportionnel au nombre de photons. Le rapport entre nombre d'ions et nombre de photons est proportionnel à la section efficace d'ionisation.

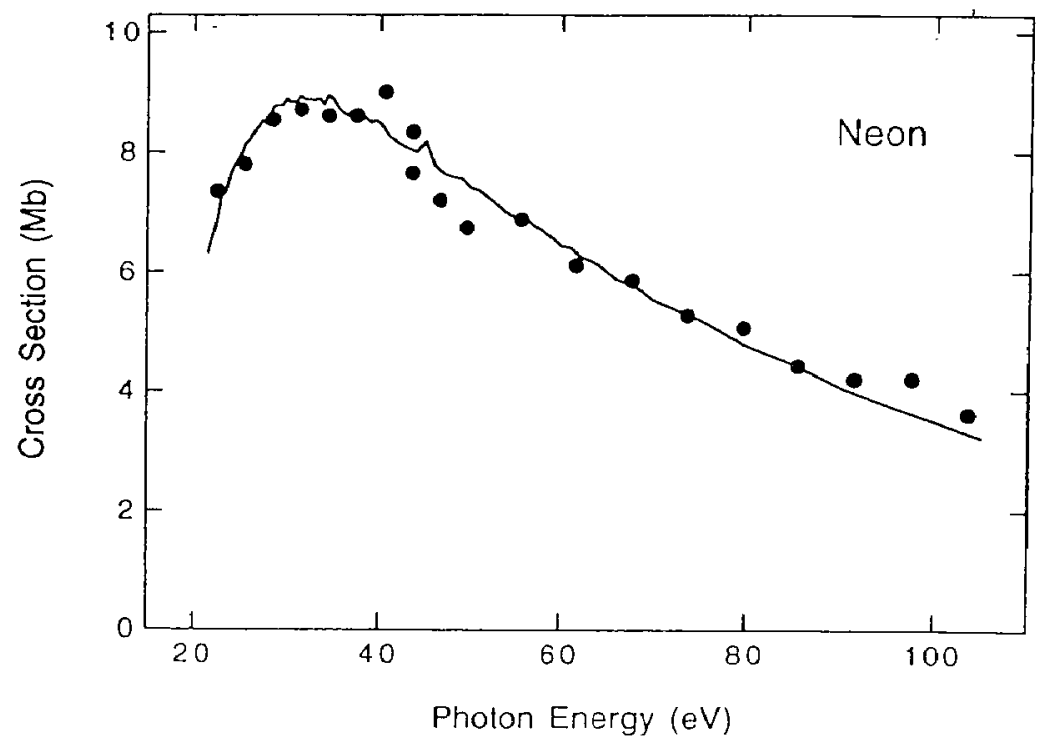

Figure 7 : Section efficace de photoionisation obtenue dans le néon.

La figure 7 montre les résultats obtenus sur le néon avec les harmoniques successives du laser LiSAF. Les points sont les résultats expérimentaux, normalisés par rapport aux données connues (en trait plein). L'accord est raisonnable, il est meilleur pour les gaz 
rares lourds où le nombre de photons disponible est plus important dans la gamme spectrale étudiée (jusqu'à $40 \mathrm{eV}$ ) et où la section efficace est plus grande, permettant donc une meilleure statistique. Notons que le nombre de photons utilisés est un facteur environ 100 fois plus petit que le nombre de photons générés, à cause de la perte sur les différentes optiques.

Nous avons également testé l'accordabilité du rayonnement harmonique à l'aide d'un laser à colorant, de durée 1 ps. Nous avons mesuré la section efficace d'ionisation du krypton à l'aide de l'harmonique 7 de ce laser, accordé par pas de $0.25 \mathrm{~nm}$ sur la gamme $590-610 \mathrm{~nm}$. On se trouve dans la région comprise entre les 2 seuils d'ionisation ${ }^{2} \mathrm{P}_{3 / 2}$ et ${ }^{2} \mathrm{P}_{1 / 2}$ du krypton, qui contient des états autoionisants de type $4 \mathrm{p}^{5} \mathrm{~ns}^{\prime}$ et nd'. Bien que notre résolution reste peu compétitive $\left(3 \times 10^{-4}\right)$, elle permet de bien décrire ces états et de retrouver les fameux profils de Fano.

A l'aide de ces deux expériences de faisabilité, nous avons démontré la versatilité et la puissance de cette nouvelle source de rayons XUV. Il est possible, soit d'explorer un grand domaine spectral en utilisant les harmoniques successives d'un laser intense, de courte durée d'impulsions, soit de réaliser des expériences de spectroscopie fine, à condition d'avoir un laser de plus longue durée et accordable. Les caractéristiques du rayonnement harmonique mentionnées ci-dessus permettent d'envisager bien d'autres applications dans des domaines différents.

1 Adresse actuelle : Laboratoire d'Electronique Quantique, Unité Associée 1202 au CNRS, Université de Rennes 1, 35042 Rennes.

\section{BIBLIOGRAPHIE}

[1] A. L'Huillier, L. A. Lompré, G. Mainfray et C. Manus, dans "Atoms in Intense Laser Fields", Ed. M. Gavrila (Advances in Atomic, Molecular and Optical Physics, Supplement 1, Academic Press, New York, 1992) p 139.

[2] Ph. Balcou, A. S. L. Gomes, C. Cornaggia, L. A. Lompré and A. L'Huillier, J. Phys. B 25, 4467 (1992).

[3] A. L'Huillier and Ph. Balcou, Phys. Rev. Lett. 70, 774 (1993).

[4] Ph. Balcou, P. Salières, K. S. Budil, T. Ditmire, M. D. Perry, A. L'Huillier, soumis à Opt. Comm.

[5] C.-G. Wahlström, J. Larsson, A. Persson, T. Starcszewski, S. Svanberg, P. Salières, Ph. Balcou and A. L'Huillier, Phys. Rev. A 48, 4709 (1993).

[6] J. L. Krause, K. J. Schafer and K. C. Kulander, Phys. Rev. Lett. 683535 (1992).

[7] A. L'Huillier, M. Lewenstein, Ph. Balcou, P. Salières, M. Y. Ivanov, J. Larsson and C.-G. Wahlström, Phys. Rev. A 48, R3433 (1993).

[8] P. Salières, T. Ditmire, K. S. Budil, M. D. Perry and A. L'Huillier, J. Phys. B 27, L2 17 (1994). 